\title{
ERRATUM
}

\section{Variable Dependencies of Quantified CSPs}

\author{
Marko Samer \\ Department of Computer Science \\ TU Darmstadt, Germany \\ samer@cs.tu-darmstadt.de
}

I. Cervesato, H. Veith, and A. Voronkov (Eds.): LPAR 2008, LNCS 5330, pp. 512-527, 2008.

(C) Springer-Verlag Berlin Heidelberg 2008

DOI 10.1007/978-3-540-89439-1_49

Reference 15 was printed incorrectly in the original version. The correct version is: Samer, M., Szeider, S.: Backdoor sets of quantified boolean formulas. (submitted for publication). Preliminary version published in: Marques-Silva, J., Sakallah, K.A. (eds.) SAT 2007. LNCS, vol. 4501, pp. 230-243. Springer, Heidelberg (2007). 